\title{
EDITORIAL
}

\section{Married to our gut microbiota}

F or richer for poorer, in sickness and in health. Our relationship with our microbiota-the vast array of microorganisms that live on or inside the human body-lasts a lifetime. Arguably, the highest density of and diversity in these microorganisms is in our gut, which is home to trillions of bacteria. The past few years has seen a surge in the number of research initiatives into the association between the gut microbiota and our health, with an ever-growing number of disorders now linked to dysbiosis of the resident microbiota, including, among others, gastrointestinal diseases such as IBD, cancer, cardiovascular disease and obesity (as well as NAFLD). In recognition of the interest in this new field as it rapidly evolves, Nature Reviews Gastroenterology \& Hepatology commissioned this special Focus issue on the gut microbiota to highlight the latest research, translate these findings into clinical practice and set the stage for future developments.

We are not just on 'friendly' terms with our gut bacteria - the relationship is infinitely more intimate than that-we are married to them. As Erika Isolauri and colleagues discuss in their Review, we are colonized by bacteria from birth, and potentially in utero. Indeed, our mothers play a vital part in providing the first 'building blocks' for the development of our resident microbiota, jump-starting the stepwise colonization of our intestines and the rest of the human body through skin-toskin contact and breastfeeding. Once the gut microbiota becomes established and stabilized by age $2-3$ years, these bacteria have a crucial role in nutrition and health. As Harry Flint and co-workers explain, the benefits of these microorganisms to health are often undervalued; they have a fundamental role in synthesizing vitamins and in helping to breakdown nondigestible products that provide energy to the human body.

This partnership between the gut and its microbiota is not always so harmonious, and a number of studies have focused on the influence that these bacteria have on disease. As Francisco Guarner et al. describe, dysbiosis of the gut bacteria has been directly linked with the development of IBD. Moreover, increasing evidence in mice and humans has shown that the gut microbiota is intrinsically linked with our metabolic health, particularly in obese individuals. With this in mind, Karine Clement, Judith Aron-Wisnewsky and Joel Doré consider how bariatric surgery might disrupt our intestinal microbiota, given that the ecology of the gut is dramatically changed by this treatment for obesity.

The challenge ahead of us, as Fergus Shanahan points out in his Perspectives article, is how we can translate

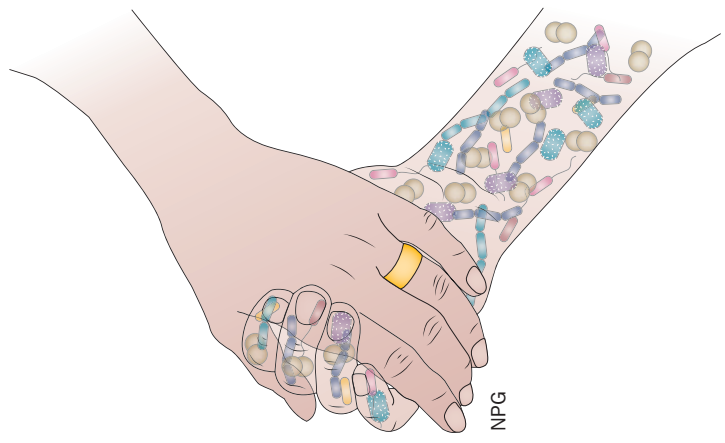

21...are we more microbe than man? 7

these new research findings into clinical practice. With better understanding of the microorganisms in our gut, one hope is that their manipulation might prove to be a future targeted therapy for a number of conditions.

Outnumbered (on a cellular level alone) by our microbial 'mates' by 10 to 1 , a question arises: are we more microbe than man? Increasingly, it seems that the gut microbiota can be considered as a human microbial 'organ'. From an ecological point of view, it could be argued that humans are a superorganism, a communal collective of human and microbial cells working as one. Thus, as medicine moves towards taking a personalized approach, should we be tailoring treatment to both our human and microbial 'selves'?

How best to manage our microbiota, however, is hotly debated. Probiotics are well established in the public conscious, with many a yoghurt advert extolling the virtues of such 'good bacteria', but their use as a medical therapy has not yet been approved by the FDA nor the European Food Safety Authority. If we can get over the 'yuck factor', then perhaps faecal microbiota transplantation may prove useful. However, much work is needed, particularly in characterizing the microbes present in the administered material and their potential long-term effects, before we are likely to see its widespread prescription in medicine; it may well transpire that faecal transplantation will be legislated as with any other form of organ donation, with careful regulation of both the donor and recipient.

As research into the microbiota continues to evolve at an unprecedented pace, we are gaining vital insights into the gut-microbiota relationship in human health and disease. We at Nature Reviews Gastroenterology \& Hepatology look forward to the exciting times ahead for the field and believe that hand in hand with our microbiota we are entering a new scientific era.

doi:10.1038/nrgastro.2012.165
Katrina Ray is Acting Chief Editor of Nature Reviews Gastroenterology \& Hepatology.

Competing interests The author declares no competing interests. 